\title{
Evaluation of the effect of inactivation by microwave and autoclave in homeopathic medicines
}

\author{
Áurea Regina Telles Pupulin, Thiago Telles Pupulin, Bruno Reis, \\ Carlos Moacir Bonato
}

\author{
State University of Maringá, Paraná, Brazil
}

\begin{abstract}
Background: The Brazilian Pharmacopoeia defines the sterilization process as a "method" intended to remove or destroy all forms of life, animal or plant, macroscopic or microscopic, saprophytic or not, present in the product concerned, without ensuring the complete inactivation of toxins or cellular enzymes. Microwaves are electromagnetic waves with frequencies ranging between $300 \mathrm{MHz}\left(300 \times 10^{6} \mathrm{~Hz}\right)$ and $300 \mathrm{GHz}\left(300 \times 10^{9}\right.$ $\mathrm{Hz}$ ) and wavelengths from $1 \mathrm{~m}$ to $1 \mathrm{~mm}[1]$. They are waves that lie within the region between TV waves and the infrared region within the spectrum of electromagnetic waves. According to the Technical Standards Textbook for Homeopathic Pharmacy, glass tubes may be reused after washed with running and purified water and inactivated by autoclaving at $120^{\circ} \mathrm{C}$ for 30 minutes or by a dry air buffer at $180^{\circ} \mathrm{C}$ for 30 minutes or at $140^{\circ} \mathrm{C}$ for 1 hour [2].
\end{abstract}

Aims: Current experiment evaluates the influence of ultra-diluted Sulphur with and without inactivation by autoclaving and microwaving for certain variables in the germination and growth of sorghum (Sorghum bicolor L. Moench - cv TX623B).

Methodology: Ten milliliters of Sulphur in homeopathic dinamizations (proposed by Hering - DH) 9DH, $18 \mathrm{DH}$ and 30DH inactivated by microwave and by autoclave heat, and control with water, were added to petri dishes in which 20 sorghum seeds were distributed. The experiment was conducted in a growth chamber (BOD) at $25^{\circ} \mathrm{C}$ and during a 16 -h photoperiod. Double-blind methodology to avoid researcher's possible interferences or trends, coupled to statistic treatment at the end of the experiment, was employed. Data underwent variance analysis and means were compared by Scott-Knott's test at $5 \%$ probability.

Results: Homeopathy Sulphur changed the evaluated parameters of 9DH, $18 \mathrm{DH}$ and $30 \mathrm{DH}$ dinamizations when compared to control (water). Differences existed with regard to effects of the different microwavetreated (M9DH, M18DH, M30DH) and autoclaving-treated (A9DH, A18DH, A30DH) dinamizations. Sulphur had no significant difference in the aerial length (CPA) when compared with that of control. In the case of the same homeopathic treatments previously undergoing autoclaving, the three dinamizations had a similar response to CPA. The same occurred with drugs submitted to microwaves. With regard to root's growth (CSR), treatment with Sulphur inhibited growth in the dinamizations $18 \mathrm{DH}$ and $30 \mathrm{DH}$ when compared to control. This did not occur with $9 \mathrm{DH}$ which had the same effect as that of water. In the case of groups treated with medicines $30 \mathrm{DH}$ and 9DH undergoing microwaves, the same effect of water was reported. Consequently, microwaves inactivated the effect of homeopathic medicine. However, in 18DH under the effect of microwaves, the effect of drug $18 \mathrm{DH}$ was reported. Consequently, there was growth inhibition but no inactivation by microwaves. In the case of groups treated with autoclaved medicine, a greater root growth was reported. This fact shows that autoclaving altered the homeopathic medicine. In fact, it had an opposite effect when 
compared to control. On the other hand, when Fresh Root Weight was evaluated, differences in control were reported only for groups treated with autoclaved medicines. These results were similar to those of the group treated with medicine $18 \mathrm{DH}$. An increase in the fresh root weight was reported agreeing with a greater root growth. Results related to the aerial part and to the root of the dry mass failed to show any significant difference among the groups.

Conclusions: Results show that different physiological responses are produced posterior to the employment of homeopathic medicine, sometimes stimulating, sometimes inhibiting root growth. The aerial part was not influenced by any treatment. On the other hand, autoclaved medicines stimulated root growth[3] [4]. This fact shows changes in the homeopathic medicine under humid heat. Microwave treatments showed an inactivation for $9 \mathrm{DH}$ and $30 \mathrm{DH}$, but not for $18 \mathrm{DH}$. The latter had the same results as those treated with Sulphur 18DH. The above results suggest that microwaves may inactivate homeopathic medicines, albeit not entirely. Therefore, it may not be a reliable inactivation methodology.

Keywords: homeopathy; seed dynamics; microwave; autoclave.

References:

[1] . Black SS. Desinfection, Sterilization and Preservation 10th Edition-1991.

[2] Manual de Normas Técnicas para Farmácia Homeopática: ampliação dos aspectos técnicos e práticos para farmácia homeopática. 3. Ed. Curitiba, 2003.

[3]. Beniter N A. Ação do medicamento homeopático. Revista Agropecuária, Ano IV, no 19, Jun/Jul, 2003.

[4] Bonato CM., SILVA EP. Effect of the homeopathic solution Sulphur on the growth and productivity of radish. Acta scientiarum agronomy, v. 25, n. 2, 2003.

\section{Avaliação do efeito da inativação por microondas e autoclave em medicamentos homeopáticos}

\section{RESUMO}

Introdução: A Farmacopéia Brasileira define o processo de esterilização como "Método" e tem por finalidade remover ou destruir todas as formas de vida, animal ou vegetal, macroscópicas ou microscópicas, saprófitos ou não, presentes no produto considerado, sem garantir a inativação completa de toxinas ou enzimas celulares. Chamamos de microondas as ondas eletromagnéticas com freqüências desde $300 \mathrm{MHZ}\left(300 \times 10^{6} \mathrm{~Hz}\right)$ até 300 $\mathrm{GHz}\left(300 \mathrm{x} 10^{6} \mathrm{~Hz}\right)$ e comprimentos de onda desde $1 \mathrm{~m}$ até $1 \mathrm{~mm}$ [1]. São, portanto, ondas que estão entre a região de ondas de TV e a região do infravermelho no espectro das ondas eletromagnéticas. Segundo o Manual de Normas Técnicas para Farmácia Homeopática os vidros podem ser reutilizados desde que lavados com água corrente e purificada e inativados através de autoclave numa temperatura de $120^{\circ} \mathrm{C} 1 \mathrm{~atm}$ por 30 minutos ou em estufa de ar seco na temperatura de $180^{\circ} \mathrm{C}$ por 30 minutos ou $140^{\circ} \mathrm{C}$ por uma hora[2].

Objetivo: Avaliar a influência do medicamento Sulphur ultradiluido com e sem inativação em autoclave e microondas para algumas variáveis na germinação e crescimento de sorgo (Sorghum bicolor L. Moench TX623B cv). 
Metodologia: Dez mililitros do medicamento Sulphur em dinamizações homeopáticas (DH-proposto por Hering) 9DH, $18 \mathrm{DH}$ e 30DH inativados pelo efeito de microondas e por calor em autoclave, e um controle contendo água foram adicionados para placas de Petri com subseqüente distribuição de 20 sementes de sorgo. $\mathrm{O}$ experimento foi conduzido em câmara de crescimento (BOD) a $25^{\circ} \mathrm{C}$ e um fotoperíodo de $16 \mathrm{~h}$. A metodologia adotada foi a do duplo-cego, para evitar possíveis interferências ou tendências por parte do pesquisador com tratamento estatístico no final do experimento. Os dados obtidos foram submetidos à análise de variância e as médias comparadas pelo teste Scott-Knott a $5 \%$ de probabilidade.

Resultados: A homeopatia Sulphur alterou os parâmetros avaliados nas dinamizações de 9DH, 18 DH e 30 DH quando comparadas ao controle (água). Ainda houve diferença quanto ao efeito das diferentes dinamizações tratadas com as microondas (M9DH, M18DH, M30DH) e com autoclavação (A9DH, A18DH, A30DH). O Sulphur no comprimento da parte aérea (CPA) não apresentou diferença significativa quando comparado com o controle. Com relação aos mesmos tratamentos homeopáticos, mas submetidos previamente a autoclavação observamos para as três dinamizações testadas o mesmo padrão de resposta para CPA, assim como os medicamentos submetidos à ação das microondas. Com relação ao crescimento da raiz (CSR) observamos para os tratamentos com medicamento Sulphur inibição do crescimento em relação ao controle nas dinamizações $18 \mathrm{DH}$ e $30 \mathrm{DH}$, mas não para $9 \mathrm{DH}$ que apresentou o mesmo efeito da água. Já para os grupos tratados com medicamento 30DH e 9DH submetido à ação das microondas observamos o mesmo efeito da água, ou seja, a microonda inativou o efeito do medicamento homeopático. Porém para o medicamento na potencia $18 \mathrm{DH}$ submetido às microondas observamos o mesmo efeito do medicamento 18DH, ou seja, inibição do crescimento e não inativação pelas microondas. Nos grupos tratados com medicamento submetido á autoclavação observamos um maior crescimento das raízes mostrando que a autoclavação alterou o medicamento homeopático passando este a ter um efeito oposto quando comparado ao controle. Por outro lado na avaliação da Massa Fresca da Raiz (MFSR) observamos diferenças do controle apenas para os grupos tratados com medicamentos submetidos á autoclavação, porem estes resultados foram iguais ao grupo tratado com medicamento 18DH. Nestes grupos houve um aumento da massa fresca da raiz, resultado este concordante com o maior crescimento da raiz observado. Os resultados da Massa Seca da Parte Aérea e da Raiz não mostraram diferenças significativas entre os grupos.

Conclusões: Os resultados observados constatam que diferentes respostas fisiológicas são geradas após a utilização do medicamento homeopático, ora estimulando, ora inibindo o crescimento do sistema radicular[3][4]. A parte área não foi afetada por nenhum tratamento. Por outro lado os medicamentos submetidos á autoclavação mostraram estimular o crescimento radicular, fato este que evidencia uma alteração do medicamento homeopático por efeito do calor úmido. Para os tratamentos com as microondas mostraram uma inativação para 9DH e 30DH, mas não para $18 \mathrm{DH}$ que mostrou resultados iguais ao tratamento com Sulphur 18DH, estes resultados sugerem que microondas podem inativar medicamentos homeopáticos, mas não totalmente, portanto não pode ser considerada uma metodologia de inativação confiável.

Palavras-chave: homeopatia, crescimento de sementes, microondas, autoclave

\section{(c)) BY-NC-ND Licensed to GIRI}

Support: The authors declare that this study received no funding.

Conflict of interest: The authors declare no conflict of interest exists.

Correspondence author: Áurea Regina T Pupulin, artpupulin@uem.br

How to cite this article: Pupulin ART, Pupulin TT, Reis B , Bonato CM. Evaluation of the effect of inactivation by microwave and autoclave in homeopathic medicines. Int J High Dilution Res [online]. 2011 [cited YYYY Month dd]; 10(36): 226-228. Proceedings of the XXV GIRI Symposium and VIII CBFH; 2011 Sep 04-07; Foz do Iguaçu (Brazil). GIRI and ABFH; 2011; Available at http://www.feg.unesp.br/ ojs/index.php/ijhdr/article/view/514/530 